\title{
Enhance the Information Content of Medical Image by Image Fusion Techniques - A Survey
}

\author{
Sumit Narayan Jarholiya1, Dr. Shachi Awasthi ${ }^{2}$ \\ ${ }^{1}$ Research Scholar,Jayoti Vidyapeeth Women's University, Jaipur, India \\ ${ }^{2}$ Faculty of Engineering and Technology, Jayoti Vidhyapeeth Women's University, Jaipur, India
}

\begin{abstract}
Image fusion is a process of combining a set of images of the same scene into one composite image. The main objective of this technique is to obtain an image that is more suitable for visual perception. At present, there are three types of fusion operators for wavelet image fusion: pixel, area and region. Image fusion has been used in many application areas such as Remote sensing, Astronomy, Multi sensor, Medical Imaging etc. Fusion is used to achieve high spatial and spectral resolutions by combining images from two sensors, one of which has high spatial resolution and the other one high spectral resolution. Numerous fusion applications have appeared in medical imaging like simultaneous evaluation of CT, MRI, and / or PET images.
\end{abstract}

Keywords: Medical Image Fusion, Wavelet Transform, Discrete Wavelet Transform (DWT ), Principal Component Analyses ( PCA), CT, MRI, and/or PET images

\section{Introduction of Image Fusion}

Image fusion is a process of combining a set of images of the same scene into one composite image. The main objective of this technique is to obtain an image that is more suitable for visual perception.

The goal of image fusion (IF) is to integrate complementary multisensor, multiview and multitemporal information into one new image containing information the quality of which cannot be achieved otherwise. The term quality, its meaning and measurement depend on the particular application. [1]

At present, there are three types of fusion operators are used for wavelet image fusion: pixel, area and region. Pixel operators can quickly get a fused image, but the image can be blurred. Area operators consider the neighboring gray value, which can reduce the edge's sensitivity and the fused image has better vision characteristics. Region operators should operate image segment to source image. Though it can obtain an image with the best vision characteristics, the speed is very slow, so it is not suitable for medical image fusion, as medical image fusion should consider the time cost. So the aim of the study is to find a proper fusion operator for medical image fusion that considers not only the time cost but also the quality of the fused image [4]. For fulfilling the aim of image fusion area operator will be used.

Image fusion has been used in many application areas like remote sensing, astronomy, multisensor and medical imaging etc. Numerous fusion applications have appeared in medical imaging like simultaneous evaluation of CT, MRI, and/or PET images. [1]

Medical image fusion is a technique in which useful information from two or more registered medical images is integrated into a new image that can be used to make clinical diagnosis and treatment more accurate. At present, the main focus of medical image fusion is on the anatomic image and the functional image, such as CT/PET, MRI/PET. [4]
Typically, the field of medical image analysis is divided into six categories:

- Post-acquisition: Preprocessing techniques such as denoising and restoration are used to restore the images so that they can be used for diagnosis.

- Segmentation: Images such as brain MRI or abdomen CT scan contain multiple features (organs). Delineating features of interest is important for analysis and accurate diagnosis.

- Registration: In computer assisted surgery, it is required to register or align the captured image with a model or a previously captured image.

- Computation: Physical quantity derivation and other computation such as fusion and compression are also required in several computer assisted therapy.

- Visualization: It is important to display medical images on screen so that a medical professional can diagnose diseases.

- Security: Personal medical health-care information is very sensitive and it is very important to secure it using techniques such as watermarking so that only legitimate user can access it and also accurately associate with the medical record with the correct patient. [5]

Another approach of Medical imaging is Image guided interventions and therapy (IGIT). The minimally invasive nature of Image guided interventions and therapy (IGIT) is often preferred over open surgeries because less trauma to the patient's body is caused, which generally is associated with easier and faster recovery. Interventional radiologists and surgeons are also becoming more experienced and comfortable in performing these procedures. The minimally invasive interventional clinicians use instruments such as needles and catheters to perform the diagnostic and therapeutic procedures, which are guided by imaging equipment. [7]

\section{Image Fusion Methods}

There are many image fusion methods that can be used to

Volume 5 Issue 9, September 2016

www.ijsr.net

Licensed Under Creative Commons Attribution CC BY 


\section{International Journal of Science and Research (IJSR) \\ ISSN (Online): 2319-7064}

Index Copernicus Value (2013): 6.14 | Impact Factor (2015): 6.391

produce high-resolution multispectral images from a highresolution panchromatic image and low-resolution multispectral images. Starting from the physical principle of image formation, this paper presents a comprehensive framework. [8]

The term CBIR (content-based image retrieval) designates the retrieval of images based on their visual similarity to a user-supplied query image or to a set of user-specified image features. CBIR can be widely used in many areas, such as photograph archives, medical diagnosis, the military, retail catalogs, and remote sensing systems. [10]

There are so many image fusion methods are available. Some of these image fusion methods are as follows:-

- Wavelet Transform Fusion

- Discrete Wavelet Transform ( DWT )

- Image Fusion Based On Wavelet Decomposition

- Pixel Based Wavelet Transform Fusion

- The Dual Tree Complex Wavelet Transform (DTCWT)

- Fusion of Multimodal Brain Images using RDWT

- Principal Component Analyses ( PCA)

\section{Wavelet Transform Fusion}

In all wavelet based image fusion schemes the wavelet transforms $\mathrm{W}$ of the two registered input images $I_{1}$ and $I_{2}$ are computed and these transforms are combined using some kind of fusion rule. Fig1 Then the inverse wavelet transform $\mathrm{W}^{-1}$ is computed and the fused image $\mathrm{I}_{1}$ is reconstructed Wavelet transform fusion schemes over several advantages over similar pyramid based fusion schemes when it comes to image fusion a the wavelet transform provides directional information while the pyramid representation doesn't introduce any spatial orientation in the decomposition process in pyramid based image fusion the fused images often contain blocking effects in the regions where the input images are significantly different .[3]

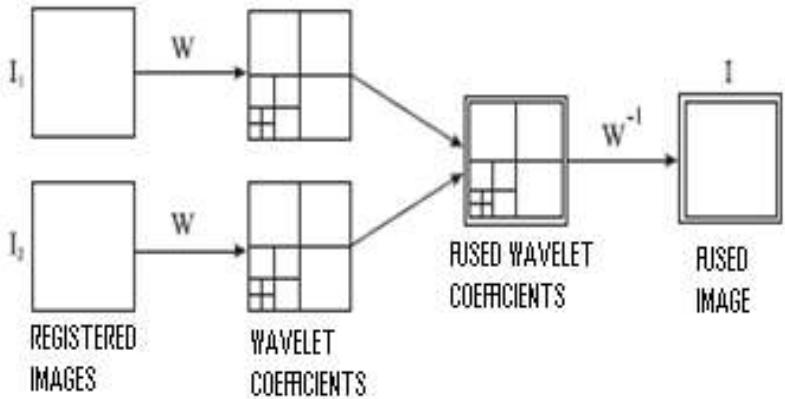

Figure 1: Fusion of the wavelet transforms of two images

\section{Discrete Wavelet Transform (DWT)}

The Discrete Wavelet Transform (DWT), which is based on sub-band coding is found to yield a fast computation of Wavelet Transform. It is easy to implement and reduces the computation time and resources required.

The discrete wavelet transform DWT is a spatial frequency decomposition that provides a flexible multi resolution analysis of an image in one dimension. The aim of the wavelet transform is to represent the signal as a superposition of wavelets, If a discrete signal is represented by $\mathrm{f}(\mathrm{t})$ its wavelet decomposition is then given by

$$
f(t)=\sum_{m, n} c_{m, n} \psi_{m, n}(t),
$$

where $\mathrm{m}, \mathrm{n}(\mathrm{t})$ is the dilated and or translated version of the mother wavelet given by the equation

$$
\psi_{m, n}(t)=2^{-m / 2} \psi\left[2^{-m} t-n\right], \quad\{2\}
$$

where $\mathrm{m}$ and $\mathrm{n}$ are integers This ensures that the signal is decomposed into normalised wavelets at octave scales .

In CWT, the signals are analyzed using a set of basic functions which relate to each other by simple scaling and translation. In the case of DWT, a time-scale representation of the digital signal is obtained using digital filtering techniques. The signal to be analyzed is passed through filters with different cutoff frequencies at different scales.

\section{Image Fusion Based On Wavelet Decomposition}

Wavelet transforms are useful to extract local details from "non-stationary" signals. DWT is based on sub-band coding and normally used for multi-resolution analysis. In DWT [2] the time is not discrete but the translation and the scale steps are discrete. The CWT (continuous wavelet transform) is defined as:

$$
W_{v} f(\mathrm{a}, b)=|a|^{-1 / 2} \int f(t) \mathrm{Wf}((\mathrm{t}-\mathrm{b}) / \mathrm{a}) \mathrm{dt}
$$

The functions $\mathrm{W}$ are called wavelets.

Here HH,HL,LH are the horizontal , vertical and diagonal detail coefficients and LL is the approximate coefficient . [2]

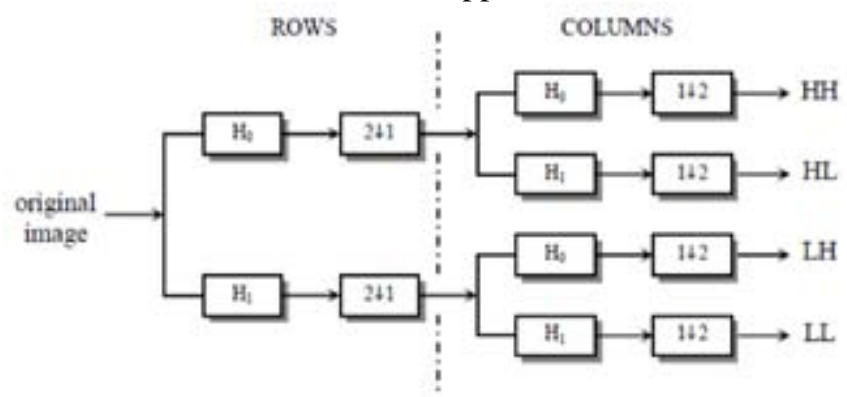

Figure 2: Image Decomposition

\begin{tabular}{|c|c|c|}
\hline $\mathbf{L I}{ }^{2}$ & $\mathrm{HL}^{2}$ & \multirow{2}{*}{ HI $^{1}$} \\
\hline $\mathrm{LH}^{2}$ & $\mathrm{HH}^{2}$ & \\
\hline & L. $H^{1}$ & $H H^{1}$ \\
\hline
\end{tabular}

The fusion process is performed by combining the detail and approximate coefficient as follows:

\section{Pixel Based Wavelet Transform Fusion}

In the case of pixel based wavelet transform fusion all respective wavelet coefficients from the input images are combined using the fusion rule Since wavelet coefficients having large absolute values contain the information about the salient features of the images such as edges and lines a good fusion rule is to take the maximum of the absolute values of the corresponding wavelet coefficients.[3] A more advanced area based selection rule is proposed in the maximum absolute value within a window is used as an 


\section{International Journal of Science and Research (IJSR) \\ ISSN (Online): 2319-7064 \\ Index Copernicus Value (2013): 6.14 | Impact Factor (2015): 6.391}

activity measure of the central pixel of the window . A binary decision map of the same size as the WT is constructed to record the selection results based on a maximum selection rule.

\section{The Dual Tree Complex Wavelet Transform (DTCWT)}

The dual tree complex wavelet transform (DTCWT) iteratively applies separable spatial filters to produce frequency sub bands in a similar way to the classic discrete wavelet transform. The prime motivation for producing the dual tree complex wavelet transform was shift invariance. In normal wavelet decomposition small shifts of the input signal are able to move energy between output sub bands. This is a result of the sub sampling necessary for critical decimation. The shift invariant discrete wavelet transform (SIDWT) was an initial attempt to integrate shift invariance into a DWT by discarding all sub sampling .The SIDWT is therefore considerably over complete [3]. It has been used for image fusion as reported in Shift invariance can also be achieved in a DWT by doubling the sampling rate .This is effected in the DTCWT by eliminating the down sampling by after the first level of filtering. Two fully decimated trees are then produced by down sampling effected by taking first the even and then the odd samples after the first level filters. To get uniform intervals between the two trees samples the subsequent filters in one tree must have delays that are half a sample different. For linear phase this is enforced if the filters in one tree are even and the filters in the other are odd. Additionally better symmetry is achieved if each tree uses odd and even filters alternately from level to level. The filters are chosen from a perfect reconstruction biorthogonal set and the impulse responses can be considered as the real and imaginary parts of a complex wavelet. Application to images is achieved by separable complex filtering. The dual tree complex wavelet transform (DTCWT) iteratively applies separable spatial filters to produce frequency sub bands in a similar way to the classic discrete wavelet transform.

\section{Fusion of Multimodal Brain Images using RDWT}

Medical images captured at different time instances can have variations due to geometric deformations. To optimally fuse two 2D/3D medical images (e.g. T1 and T2 brain images), we first need to minimize linear and non-linear differences between them using registration technique. Medical image registration is about determining geometrical transformation that aligns points in one medical data set with corresponding points in another data set [5]. We first propose mutual information based non-linear registration algorithm for registering multimodal medical images. Mutual information is a concept from information theory in which statistical dependence is measured between two random variables. Registration algorithm of medical images is described as follows:

Let I1 and I2 be the input multimodal brain images for registration. Mutual information between the two images can be represented as,[5]

$$
\mathrm{M}(\mathrm{I} 1, \mathrm{I} 2)=\mathrm{H}(\mathrm{I} 1)+\mathrm{H}(\mathrm{I} 2)-\mathrm{H}(\mathrm{I} 1, \mathrm{I} 2)
$$

$\mathrm{H}(\cdot)$ is the entropy of the image and $\mathrm{H}(\mathrm{I} 1, \mathrm{I} 2)$ is the joint entropy. Registering I1 with respect to I2 requires maximization of mutual information between I2 and I1, thus maximizing the entropy $\mathrm{H}(\mathrm{I} 1)$ and $\mathrm{H}(\mathrm{I} 2)$, and minimizing the joint entropy $\mathrm{H}(\mathrm{I} 1, \mathrm{I} 2)$. Further, normalized mutual information can be written as,[5]

$$
\mathrm{NM}(\mathrm{I} 1, \mathrm{I} 2)=[\{\mathrm{H}(\mathrm{I} 1)+\mathrm{H}(\mathrm{I} 2)\} / \mathrm{H}(\mathrm{I} 1, \mathrm{I} 2)]\{6\}
$$

The registration is performed on a transformation space, $S$, such that

$$
S=\left[\begin{array}{lll}
a & b & 0 \\
c & d & 0 \\
e & f & 1
\end{array}\right]
$$

where, $a, b, c, d$ are shear, scale, and rotation parameters, and $e, f$ are the translation parameters. Using the normalized mutual information, we define a search strategy to find the transformation parameters, $S$, by exploring the search space, $S$.

$$
\mathrm{S}=\arg \max \{\mathrm{S}\}\{\mathrm{NM}(\mathrm{I} 2, \mathrm{~S}(\mathrm{I} 1))\}\{7\}
$$

Finally, IRDWT is applied on the four fused sub bands to generate the fused medical image $I F$.

$$
\mathrm{IF}=\mathrm{IRDWT}(\mathrm{IaF}, \mathrm{IvF}, \mathrm{IdF}, \mathrm{IhF})\{8\}
$$

This process is represented in by the diagrams given below.

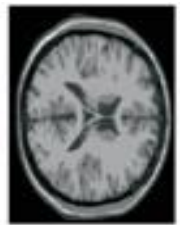

Souroe Image

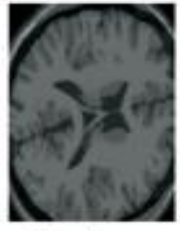

Target irage

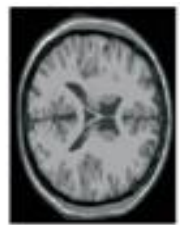

Souroe Image

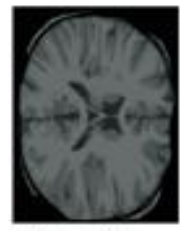

Registered Image

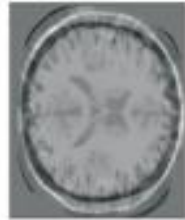

Otference of source and target image

Dference of registered and target image

Figure 3: Example of multimodal brain image registration

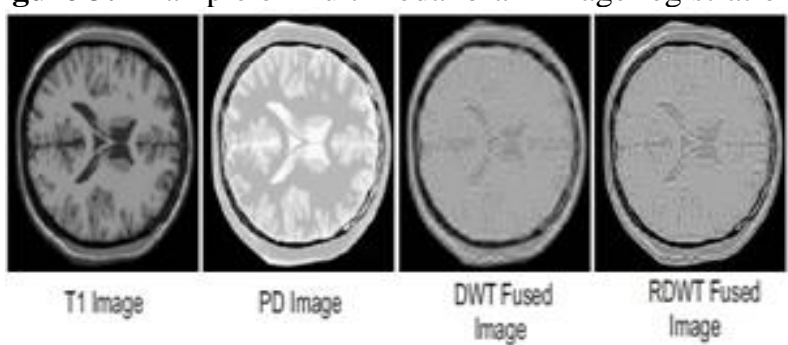

Figure 4: Fusion of T1 and PD images using the RDWT fusion algorithm

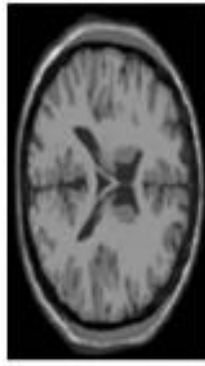

T1 mage

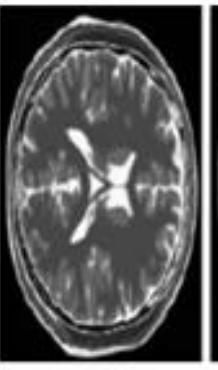

T2/mage
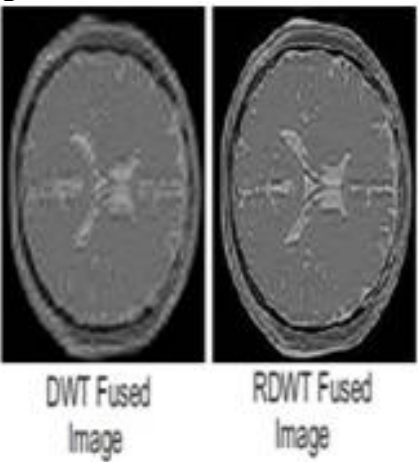

Figure 5: Fusion of T1 and T2 images using DWT and RDWT fusion algorithms

\section{Volume 5 Issue 9, September 2016




\section{International Journal of Science and Research (IJSR) \\ ISSN (Online): 2319-7064 \\ Index Copernicus Value (2013): 6.14 | Impact Factor (2015): 6.391}

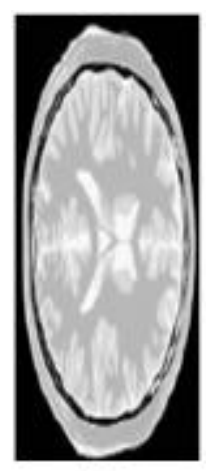

PO maye

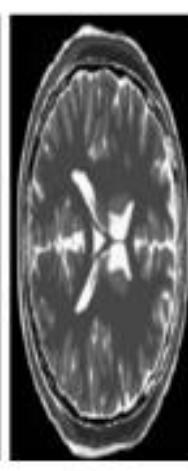

T2 1 mạe

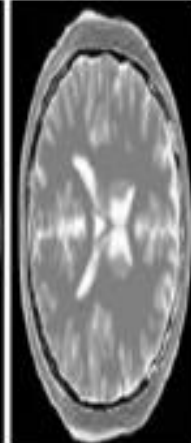

DNTFued

mase

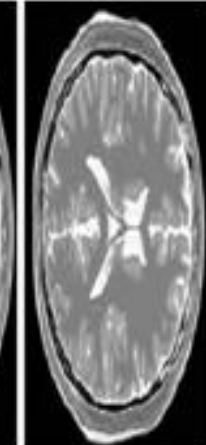

RDVIFived
Figure 6: Fusion of T2 and PD images using DWT and RDWT fusion algorithms

\section{Principal Component Analyses (PCA)}

The PCA is a statistical technique which is used to transform the multivariate dataset of correlated variables into a dataset of uncorrelated linear combinations of the original variables. [9] There's involvement of mathematical formula for change of factors which can be called key components. It computes a tight and optimum explanation of the info set. The very first key aspect corresponds to the maximum amount of difference probable in the info and every subsequent aspect corresponds to the rest of the variance. First key aspect is taken across the direction of optimum variance. The 2 nd key aspect is limited to lay in the subspace at a 90 level angle of the first. Within this Subspace, this aspect items the direction of optimum variance. The next key aspect is taken in the optimum difference direction in the subspace at a 90 level angle to the former two. [6]

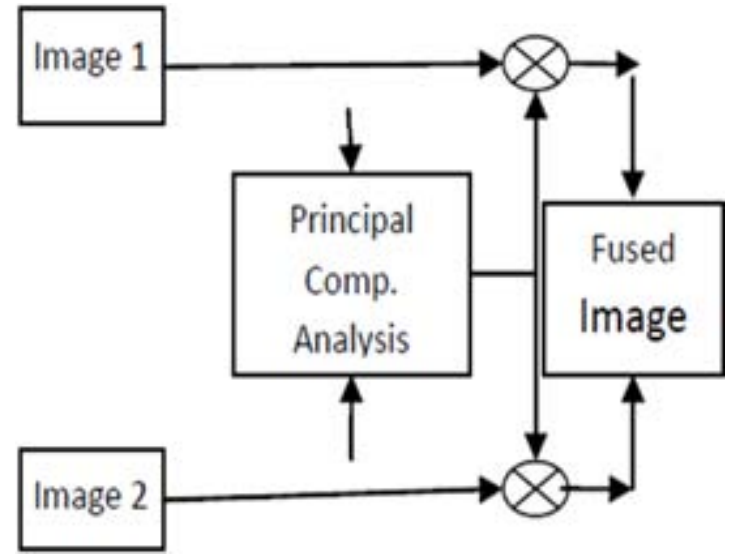

Figure 7: Image fusion process using PCA

\section{Conclusion}

The fusion rules and fusion operators are very important for image fusion, so it is preferable to select fusion rules and fusion operators according to the aim of the image fusion. The window image fusion rules and area fusion rules operator, also called window fusion rules, will be used in order to make the fusion image more in accord with human visual characteristics. For the low frequency wavelet coefficients, as it's the original smooth image, it contains the most information from the original image and for the high frequency wavelet coefficients, we used local standard deviation larger to detect edge information mutations.
Finally, through the consistency check, we got the fusion wavelet coefficients. On the whole, using the proposed fusion rules, we can get a high performance fusion image.

\section{References}

[1] Jan Flusser, Filip Sroubek, and Barbara Zitov'a, “ Image Fusion: Principles, Methods, and Applications", Institute of Information Theory and Automation ,Academy of Sciences of the Czech Republic (2007)

[2] Madhusmita Sahoo, “ Biomedical Image Fusion \&Segmentation using GLCM ", IJCA Special Issue on "2nd National Conference- Computing, Communication and Sensor Network" CCSN, pp 34-39,2011

[3] Stavri Nikolov, Paul Hill, David Bull, Nishan Canagarajah, "Wavelets For Imaged Fusion", Image Communications Group Centre for Communications Research,University of Bristol

[4] Zhi-hai Xu,Ling-xiang Liu, Lei Tong, Lin-hong Zhou, Chao-min Chen, "Wavelet medical image fusion algorithm based on local area feature", Research Article/Biological and Biomedical Reports (ISSN: 21624186), 2012, 2(1), 25-31

[5] Richa Singh, Mayank Vatsa, Afzel Noore,“ Multimodal Medical Image Fusion using Redundant DiscreteWavelet Transform", West Virginia University, Morgantown, WV, USA

[6] Swathi.P.S, Sheethal.M.S, Vince Paul , "Survey on Multimodal Medical Image Fusion Techniques", IJCSET , January 2016 , Vol 6, Issue 1, ISSN:22310711, pp 33-39

[7] Dani"el Ruijters, "Multi-modal image fusion during minimally invasive treatment", Technische Universiteit Eindhoven, 2010.

[8] Zhijun Wang, Djemel Ziou, Costas Armenakis, Deren $\mathrm{Li}$, and Qingquan $\mathrm{Li}$ “A Comparative Analysis of Image Fusion Methods",IEEE TRANSACTIONS ON GEOSCIENCE AND REMOTE SENSING, VOL. 43, NO. 6, JUNE 2005

[9] M.D. Nandeesh and Dr.M. Meenakshi, "Image Fusion Algorithms for Medical Images-A Comparison ",Bonfring International Journal of Advances in Image Processing, Vol. 5, No. 3, July 2015, pp 23-26.

[10] S. Yang, I. Atmosukarto, J. Franklin, J. F. Brinkley, D. Suciu, and L. G. Shapiro, "A Model of Multimodal Fusion for Medical Applications", University of Washington

\section{Author Profile}

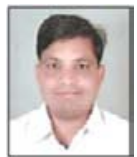

Sumit Narayan Jarholiya pursuing his Doctorate from Jayoti Vidyapeeth Women's University, Jaipur India. He did his Master of Engineering (Digital Communication) from Shri Ram Institute of Technology, Jabalpur, He had completed B. Tech. (ET \& CS ) from Mahatma Gandhi Chitrakoot Gramodaya University in 1998.

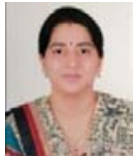

Dr. Shachi Awasthi Tiwari is awarded her Doctorate from Rani Durgavati University, Jabalpur She had Completed her Masters of Engineering ( Digital Communication ) from Shri Ram Institute of Technology, India

\section{Volume 5 Issue 9, September 2016}

\section{www.ijsr.net}

